\title{
Transcatheter aortic valve-in-stentless surgical aortic valve insertion: Hype or hope?
}

\author{
Kevin L. Greason, MD
}

See related article on pages 91-8.

Readers of the Journal will find interest in this issue's article by Duncan and colleagues ${ }^{1}$ about transcatheter aortic valve-in-valve insertion for failing surgical aortic stentless bioprosthetic valves. There is no shortage of ballyhoo promoting initial biologic surgical aortic valve replacement with planned transcatheter aortic valve-in-valve insertion bailout for subsequent surgical valve deterioration. Is that hype, or is it a hoped for effective treatment algorithm? The study in this issue applies some welcome scientific investigation to the issue.

This report is important for 3 reasons: (1) it concentrates on the specific issue of valve-in-stentless surgical aortic valve insertion; (2) it highlights the difficulty of transcatheter valve insertion in the setting of severe aortic valve regurgitation; and (3) it reports on the detailed use of the CoreValve transcatheter valve (Medtronic plc, Minneapolis, Minn).

The focus of this study is 22 high-risk patients (Society of Thoracic Surgeons predicted mortality risk of $14 \% \pm 8 \%$ ) with failing stentless surgical aortic valve prostheses: homograft in 17 patients, Toronto valve (St Jude Medical, Inc, St Paul, Minn) in 3, Freestyle valve (Medtronic) in 1, and native resuspended aortic valve in 1 . The mode of valve failure was severe aortic valve regurgitation, without any mention of valve stenosis.

Duncan and colleagues ${ }^{1}$ used multislice computed tomography to determine the aortic valve annulus size. Measurements were standard for any transcatheter aortic valve insertion assessment and included height of the coronary arteries, aortic annulus perimeter, and left ventricular outflow tract in orthogonal planes. Duncan and colleagues ${ }^{1}$ based their transcatheter valve selection on the annular perimeter.

Surgeons and cardiologists who perform transcatheter aortic valve insertion will recognize the perceived difficulty with valve-in-stentless aortic valve insertion in the setting

\footnotetext{
From the Division of Cardiovascular Surgery, Mayo Clinic, Rochester, Minn. Disclosures: Author has nothing to disclose with regard to commercial support. Received for publication March 28, 2015; accepted for publication March 30, 2015; available ahead of print May 1, 2015.

Address for reprints: Kevin L. Greason, MD, Division of Cardiovascular Surgery,

Mayo Clinic, Rochester, MN 55905 (E-mail: greason.kevin@mayo.edu).

J Thorac Cardiovasc Surg 2015;150:99-100

$0022-5223 / \$ 36.00$

Copyright (c) 2015 by The American Association for Thoracic Surgery http://dx.doi.org/10.1016/j.jtcvs.2015.03.060
}

of isolated severe aortic valve regurgitation. The struggle is twofold: (1) identifying the correct anatomic position to deploy the valve and (2) avoiding valve migration, because there is no stenosis in which to anchor the transcatheter prosthesis.

Duncan and colleagues ${ }^{1}$ report that during valve insertion fluoroscopic images were obtained coax-

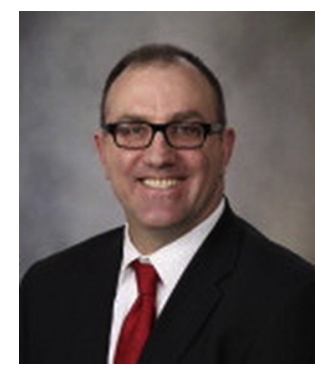
ial to the stentless aortic valve prosthesis in 19 patients with valvular calcification. There was no calcification, however, in 3 patients $(14 \%)$. In that group, the authors placed catheters in the noncoronary and left coronary sinuses to aid delineation of the target landing zone, and this is a helpful technical note. The final position of the transcatheter device was confirmed by transesophageal echocardiography before the device was fully deployed.

Duncan and colleagues ${ }^{1}$ report excellent outcomes. The coronary artery occlusion rate and 30-day mortality were both notably $0 \%$. The mean transcatheter aortic valve systolic Doppler gradient was only $12 \pm 9 \mathrm{~mm} \mathrm{Hg}$, and paravalvular regurgitation was none to mild in 19 patients and mild to moderate in 3 , with no occurrences of severe regurgitation. These levels of regurgitation were unchanged at 1year follow-up, and there were no instances of late valve migration.

There were, however, 3 cases of acute device migration and 1 of device embolization. Overall procedure success was defined as a single valve implanted in the correct anatomic location, and this was achieved in only 18 patients $(82 \%)$. The device embolization was technically related, but why the otherwise relatively low implantation success rate?

The answer may relate to inadequate transcatheter valve oversizing. Duncan and colleagues ${ }^{1}$ report that the median perimeter valve oversizing in this group of patients was only $4.7 \%$ (range $-4.9 \%$ to $24.3 \%$ ). Current experience with the CoreValve prosthesis, as reported by Dvir and colleagues, ${ }^{2}$ is that greater oversizing (ie, $>15 \%$ ) is probably indicated.

In this single-center series of high-risk patients, outcomes were excellent with respect to morbidity and mortality. It is likely that greater transcatheter valve oversizing will result in less paravalvular regurgitation and acute valve migration. The preliminary assessment is that transcatheter aortic valve-in-stentless surgical aortic valve insertion is not hype, but it is to be hoped a useful paradigm. 


\section{References}

1. Duncan A, Davies S, Di Mario C, Moat N. Valve-in-valve transcatheter aortic valve implantation for failing surgical aortic stentless bioprosthetic valves: A single-center experience. J Thorac Cardiovasc Surg. 2015;150:91-8.
2. Dvir D, Webb JG, Piazza N, Blanke P, Barbanti M, Bleiziffer S, et al. Multicenter evaluation of transcatheter aortic valve replacement using either SAPIEN XT or CoreValve: degree of device oversizing by computed-tomography and clinical outcomes. Catheter Cardiovasc Interv. April 2, 2015 [Epub ahead of print]. 